\title{
Classification Algorithms of Maternal Risk Detection For Preeclampsia With Hypertension During Pregnancy Using Particle Swarm Optimization
}

\author{
Muhlis Tahir, Tessy Badriyah, Iwan Syarif \\ Departement of Information and Computer Engineering \\ Graduate Program of Engineering Technology \\ Politeknik Elektronika Negeri Surabaya \\ Jl. Raya ITS, Sukolilo, Surabaya, 60111, Indonesia \\ Telp: 62315947280 Fax: 62315946114 \\ E-Mail : muhlistahir@pasca.student.pens.ac.id, tessy@pens.ac.id, \\ iwanarif@pens.ac.id
}

\begin{abstract}
Preeclampsia is a pregnancy abnormality that develops after 20 weeks of pregnancy characterized by hypertension and proteinuria. The purpose of this research was to predict the risk of preeclampsia level in pregnant women during pregnancy process using Neural Network and Deep Learning algorithm, and compare the result of both algorithm. There are 17 parameters that taken from 1077 patient data in Haji General Hospital Surabaya and two hospitals in Makassar start on December 12th 2017 until February 12th 2018. We use particle swarm optimization (PSO) as the feature selection algorithm. This experiment shows that PSO can reduce the number of attributes from 17 to 9 attributes. Using LOO validation on the original data show that the result of Deep Learning has the accuracy of $95.12 \%$ and it give faster execution time by using the reduced dataset (eight-speed quicker than the original data performance). Beside that the accuracy of Deep Learning increased $0.56 \%$ become $95.68 \%$. Generally, PSO gave the excellent result in the significantly lowering sum attribute as long as keep and improve method and precision although lowering computational period. Deep Learning enables end-to-end framework, and only need input and output without require for tweaking the attributes or features and does not require a long time and complex systems and understanding of the deep data on computing.
\end{abstract}

Keywords : Data Mining, Preeclampsia, Feature Selection, Classification. 


\section{INTRODUCTION}

Hypertension (high blood pressure) is the most familiar medical complication knowing by mother during pregnancy. Preeclampsia (more written as pre-eclampsia) issue most serious pregnancy complication, breathtaking $4-8 \%$ of all pregnancies. It is repeated (is different to earlier disorders), hypertension syndrome, whichever, if it is not prepared, can advise into eclampsia, a very fatal and usually cause a serious condition identified by mass of thickened blood and convulsion. when we look at separating the above assertion, preeclampsia is a principal subscriber to maternal and fetal morbidity and mortality general. Preeclampsia is described to high blood pressure (hypertension), fluid retention (edema) and excess protein rate in the urine (proteinuria). This evidence is nope seen in the beginning level from pregnancy and therefore preeclampsia may be hard to identify. This can simply be detected to the routine antenatal hearing of maternal and urine blood tension, and because such female without entryway to satisfy health services are especially at risk [1].

Currently, there are no at all to cure preeclampsia. In heavy matter, the simple how to ease maternal indication is to cause artificial labor whether in premature delivery by cesarean section. Premature birth could own heavy consistency and each year four million babies are born by fetal growing limitation for a consistency of preeclampsia. Even though preeclampsia have been the point of many years of scientifical study, the correct etiology of conditions is anonymous. The recent study suggests that needy placental establishment will preclude the relocation of nutrients of mom to a baby is very important for the development of health. Through we study, our purpose, especially which lead preeclampsia and to recognize how for advance diagnosis of the condition, which allows we to supervise it over to good purpose. We trust for expanding safe and effective therapies for preeclampsia and lower the risks that inflict on mothers and children around the world [2]. So is necessary to make predictive models employ risk factors by data mining techniques to interventions relative to the expansion of preeclampsia. Health data record could it be to create a resolve for support systems and recognize diseases preeclampsia. any of there is by using data mining method that aims extract and finds a scheme of an aggregation of precious information. within the method of data mining, there are several methods of learning so that can be used to balance two methods of preeclampsia patient data, so lest in the health sectors can be used to predict Preeclampsia, each method also have a different feature model.

This study uses Preeclampsia registry data, obtained from the Hospital. We make how to certain in data mining to correct raw data, prepare to clean up data as exercise data for knowing. The beginning, we make pre-processing starting of data cleaning, data integration, and data normalization. further, we use the feature selection algorithm to grade attributes, how what a comparison of three of Data Mining [3]. the last step, we use the Data Mining method as a classification algorithm with Leave-one-out as a validation 
sampling. Further, this article will provide an analysis of the facts and knowing of the distribution of preeclampsia data[4].

\section{RELATED WORKS}

Mario W.L. Moreira et al [5] [6] [10] has made research on predicted preeclampsia using data mining. The modeling is the Bayesian network. In his research Bayesian network proposed an intelligent decision support system in predicting preeclampsia to help doctors or experts in the care of pregnant women. In this qualitative and quantitative modeling to build the network is also presented in this paper. The main contribution of this paper is a decision-making system during pregnancy care with Bayesian network modeling. A further study in classifying the severity of hypertension in pregnant women has done the comparison of Naïve Bayes modeling and AODE (Average One-Dependence Estimators Classifier) in conclusion Naïve Bayes has excellent performance with a precision of 0.400 and F-Measure of 0.397 than AODE modeling results with 0.275 and 0.295 as Precision value and F-Measure value. In the subsequent research modeling conducted on the research is Random Forest applied to pregnant women to know early disorders in pregnancy. From this modeling, the result obtained F-Measure 0.431 and ROC curve 0.731 and the value of Kappa 0.2505 than the previous modeling of preeclampsia prediction.

Eduardo Tejera, et al [7] has made his research on preeclampsia using artificial neural networks with heart rate variability index (HRV) in women with normal pregnancies, hypertension, and preeclampsia. Classification of artificial neural networks uses signals composed by time intervals between RRs generated by EKG records. In addition to the HRV index, researchers also considered other factors such as maternal history either before pregnancy or during pregnancy and the result of blood pressure, so as to obtain a sensitivity value of about $80 \%$ in pregnant women who have hypertension and normal. And the other sensitivity value is about $85-90 \%$. The conclusion shows the HRV index and Artificial Neural Network can be useful for research and characterization of pregnant women.

Souvik Saha, et al [8], has conducted research with K-Nearest Neighbor and Meta-Heuristic Algorithms algorithms with the process of resolving genes. samples of normal microarray genes and preeclampsia and a set of genes useful for preeclampsia. Complications during pregnancy cause hypertension and protein levels in urine increase. From the normal data set or response of 25000 genes (rows) that have 75 samples (columns) and we have selected 30 genes as critical genes of the disease. By optimizing with Simulate Annealing and Particle Swarm Optimization, out of 150 samples (75 with normal category and 75 categories of Pre-eclampsia) showed 80-90 samples correctly. PSO outperformed the SA with an average score of 150 samples. 
Costa K, et al [9], has made a study of artificial neural network modeling in maternal databases whose goal is to produce early predictions of preeclampsia. The study consisted of 6838 cases of pregnant women in England obtained from Harris's Copyright Research on fetal treatment in London. Of the 24 parameters obtained from the measurements and recorded 15 parameters were considered most likely to affect the characterization of preeclampsia. With feedforward neural structures, either multilayer or multislab standards are tried for prediction. The result obtained $83.6 \%$ classification and on the test result data $93.8 \%$.

Wenshuai Cheng, et al. [11] In this study, focused on gestational hypertension, in 412 pregnant women who presented risk factors to patients. The results, taking into consideration the estimated accuracy of the model, of the relatively small factors obtained by gestational hypertension and preeclampsia groups were $13.3 \%, 8 \%$, and $14.3 \%$.

\section{ORIGINALITY}

More recently, the authors have tried to include changes in some parameter markers based on literature in the form of journals, doctors Obstetrics \& Gynecology Specialist (SpOG). There are several parameters that can be used for age between 20 years or above 35 years, upgrade in body mass index, primipara gave birth for the first time), placental measure, surplus uterine distention, history of preeclampsia, history of hypertension, multiple pregnancies, and sickness that accompany pregnancy such as diabetes mellitus and obesity. This research builds a data mining approach to find out the characteristics of pregnant women as a risk factor for the occurrence of preeclampsia and to make an appropriate prediction model for preeclampsia. Of the several models that use, in the Neural Network method, there is still an accuracy value below 90\%. And Neural Network method is still not fully use the same numbers and attributes. In this study, we propose comparative comparisons with other algorithms. Using some of the previously untested attributes and data he took from RSU Haji Surabaya and 2 hospitals in Makassar. And determine the factors - factors of patients preeclampsia such as lifestyle and genetic factors. According to obstetrics and gynecology specialists are from personal data and laboratory examination. Some of the most important parameters of personal data and blood tests are blood pressure (mmHg), a number of proteinuria (mg) and previous preeclampsia factors. After designing the prediction and grade of risk by preeclampsia with extraction features the subsequent is to analyze few of the optimum methods compared. The method is developed to be used in data mining techniques and collected precious information of few information congregate about preeclampsia taken from RSU Haji Surabaya and 2 hospitals in Makassar and for predicting how advanced the chance grade of patients with Preeclampsia so helpful for midwives and doctors at RSU Haji Surabaya and 2 hospitals in Makassar and for science. 


\section{SYSTEM DESIGN}

The following, i would explain in its entirety explain the whole phase of this study by predicting preeclampsia by using feature selection. The result of calculation with feature selection by using particle swarm optimization then used for analysis of mild preeklampsia and severe preeclampsia.

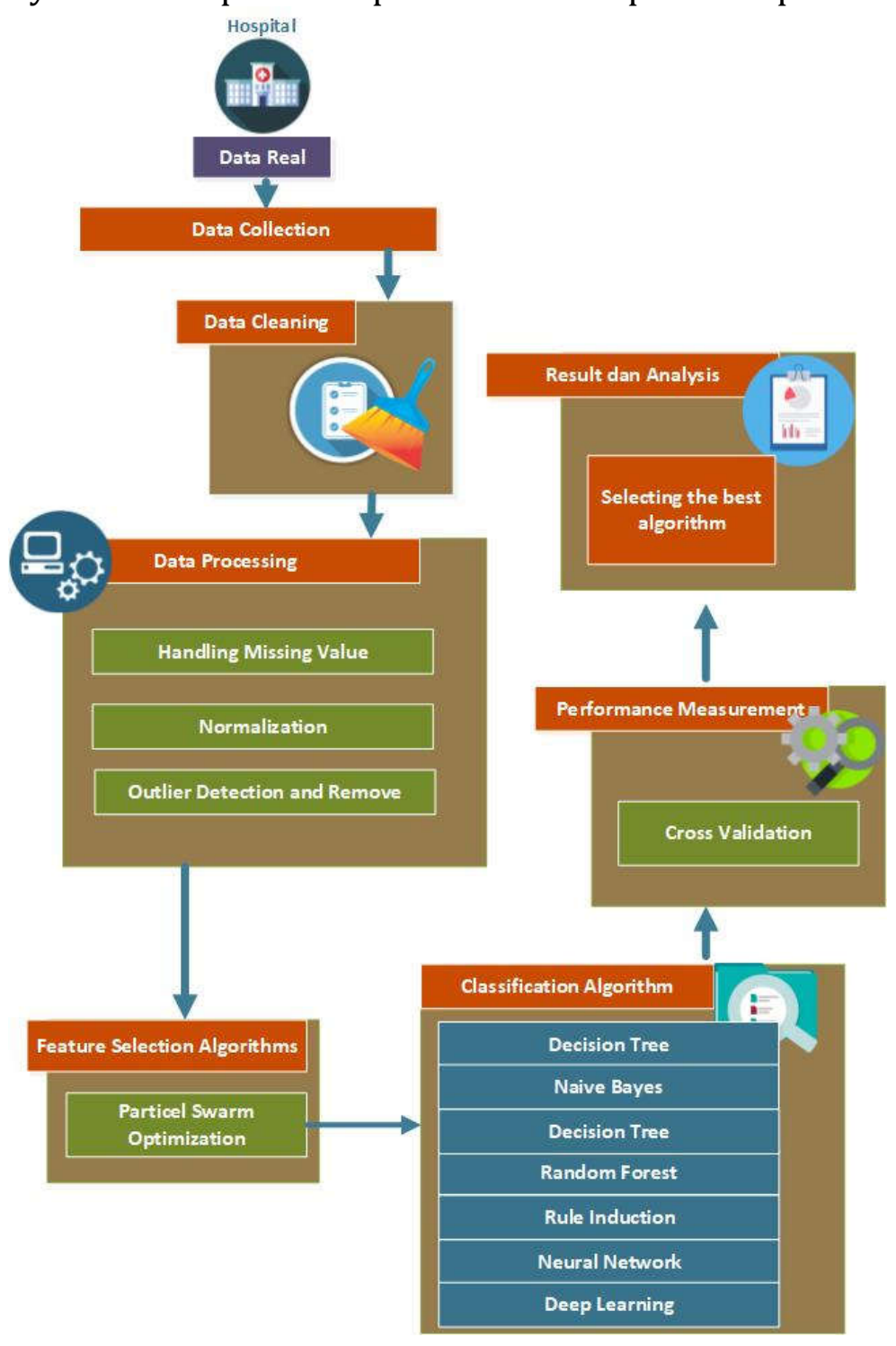

Figure 1. System Design

\subsection{Data Collection}

In study used a sample of preeclampsia from the General Hospital. The preeclampsia dataset consists of 17 attributes, 1,077 1077 sample, two classes, and data are taken through the last 2 years (2016-2017 from RSU HAJI Surabaya as many as 239 medical records) and taken from the last 4 years (2013-2017 from two Hospital in Makassar as many as 838 medical records) in this study. The preeclampsia dataset normally own two classes of 
patients, consisting of mild preeclampsia and severe preeclampsia. Further, our dataset describes the allocation of samples of pre-eclampsia patients that differ from datasets and other analysis results.

Table 1. Parameters That Were Used For Preeclampsia Prediction

\begin{tabular}{|l|}
\hline 1. RM \\
\hline 2. First Pregnancy \\
\hline 3. Age \\
\hline 4. Mean Arterial Pressure (MAP) \\
\hline 5. Systolic Blood Pressure \\
\hline 6. Dystolic Blood Pressure \\
\hline 7. BMI (Body Mass Index) \\
\hline 8. Classification BMI \\
\hline 9. Previous PE? \\
\hline 10. Personal History of Hypertension? \\
\hline 11. Personal Pregnancy History of Hypertension? \\
\hline 12. Diabetes Pragestasional? \\
\hline 13. Glukosa mg/dl \\
\hline 14. Proteinurine \\
\hline 15. Childbirth Process \\
\hline 16. Pre-exiting Medical Conditions \\
\hline 17. Mother had Mild Preeclampsia or Severe Preeclampsia? \\
\hline
\end{tabular}

\subsection{Data Cleaning}

The data to be analyzed by data mining techniques is sometimes incomplete (less than the value of a particular attribute or attribute of interest, or contains only aggregate data), contains (contains errors, or outlier values deviate from expected), and is inconsistent (eg, contains the difference in the departmental code used to categorize the goods). Data Cleaning device to "clear" the data to contents in the lost value, flattening the noise data, recognize or erase outliers, and resolving discrepancy [14].

\subsection{Data Processing}

The process of preprocessing data is to prepare data into fixed data before data becomes training data. It assigned relies at data mining experts to improve data quality, improve the precision and influence of data mining processes. The pre-processing assignment would require $60 \%$ from the data mining process attempts [15]. I follow in main effort at preprocessing data which consists Handle missing values, normalization and outlier and delete detection.

\subsection{Feature Selection}

Particles Swarm Optimization (PSO) an evolutional compute technology advanced extend by Kennedy and Eberhart in 1995 and stimulated by group bird actions to achieve goals that are not fully famous. PSO is very able, simple 
to for implement and useful computing. related different evolutionary algorithms, PSO do a search utilize populations (named swarms) of fellow (particles) that is for updated from iteration to iteration [15]. For finding the best solution, every particle changes its search direction according to two factors, its previous best experience (called the best or best experience) and the optimum impression of the entire flock (overall better or the best). The best district particles may be respected as perceptive parts whilst the optimum particles globally are well-thought-of as for social parts[16]. every particle in the category describe one likely resolution to this problem. Initially, a plume of particles was gave a disorded opening location and rate and updated according to the subsequent this equation:

$$
\begin{aligned}
& V_{i d}^{k+1}=\omega x V_{i d}^{k}+C_{1} \times \operatorname{rand}_{1} \times\left(P_{i d}-X_{i d}\right)+C_{2} \times \operatorname{rand}_{2} \times\left(\left(G_{i d}-X_{i d}\right)\right. \\
& V_{i d}^{k+1}=X_{i d}^{k+1}+V_{i d}^{k+1}
\end{aligned}
$$

Whereabout $\mathrm{x}$ is the place of the particle $\mathrm{i}, \mathrm{v}$ is its rate, $\mathrm{d}$ is the dimension, $\mathrm{k}$ is period and $\omega$ is the inertia heaviness representing how much the previous speed is maintained as it travels. $\mathrm{C}_{1}$ and $\mathrm{C}_{2}$ are knowledge factors, $\mathrm{r} 1$ and $\mathrm{r} 2$ are weighting parameters, $\mathrm{P}_{-}$id is local best whereas $\mathrm{G}_{-} \mathrm{id}$ is the broad optimum particle. The suitability of every particle is projected for each iteration, the best and individual will is too for updated using Equations 1 and 2. after the cessation criterion is reached, the PSO would own good fitness, a total of generations or convergence factors such like as a minimum threshold of population changes. The pseudocode of the PSO algorithm for

\begin{tabular}{|c|}
\hline Algorithm 2 Particle Swarm Optimization pseudo-code \\
\hline $\begin{array}{l}\text { 1. for every particle i do } \\
\text { 2. initialize place } x_{i} \text { and speed } v_{i} \\
\text { 3. end for } \\
\text { 4. while halt criteria not met do } \\
\text { 5. for all particle } i \text { do } \\
\text { 6. set personal best } x i \text { as the best place set up so far by the particle } \\
\text { 7. set global best } g \text { as best position found so far by the whole swarm } \\
\text { 8. end for } \\
\text { 9. or all particle } i \text { do } \\
\text { 10. update velocity using: } v_{i, j}^{t+1}=\omega v_{i, j}^{t}+c_{1} r_{1}\left(p_{i, j}-x_{i, j}^{t}\right)+c_{2} r_{2}\left(p_{g, j}-x_{i, j}^{t}\right) \\
\text { 11. update position using: } x_{i, j}^{t+1}=x_{i, j}^{t}+v_{i, j}^{t+1} \\
\text { 12. end for } \\
\text { 13. end while }\end{array}$ \\
\hline
\end{tabular}
feature selection is described in below [17]. 


\subsection{Classification Algorithms}

A very important part in data mining is the classification technique, that is how to study a set of data so that the resulting rules that can classify or recognize new data that has not been studied.

\subsubsection{Neural Network}

A neural network or as usual named Artificial Neural Network (ANN) is a how of mimicking nerve cell tissue in the brains. the computational trick in these way lies in the relationship among the neurons. There are at least two layers consisting of the input layer (include input value nodes) and the output layer (include output value nodes after processing inactivation function). While on a multilayered neural network, in the input layer and the output layer there is a hidden layer that contains the output value nodes that are used as input values at other nodes [18]. Modeling a neuron in this method is as follows:

$\mathrm{Z}=\mathbf{a}_{1} \mathbf{w}_{1}+\mathbf{a}_{2} \mathbf{W}_{2}+\ldots+\mathbf{a}_{k} \mathbf{W}_{k}+\mathbf{b}$

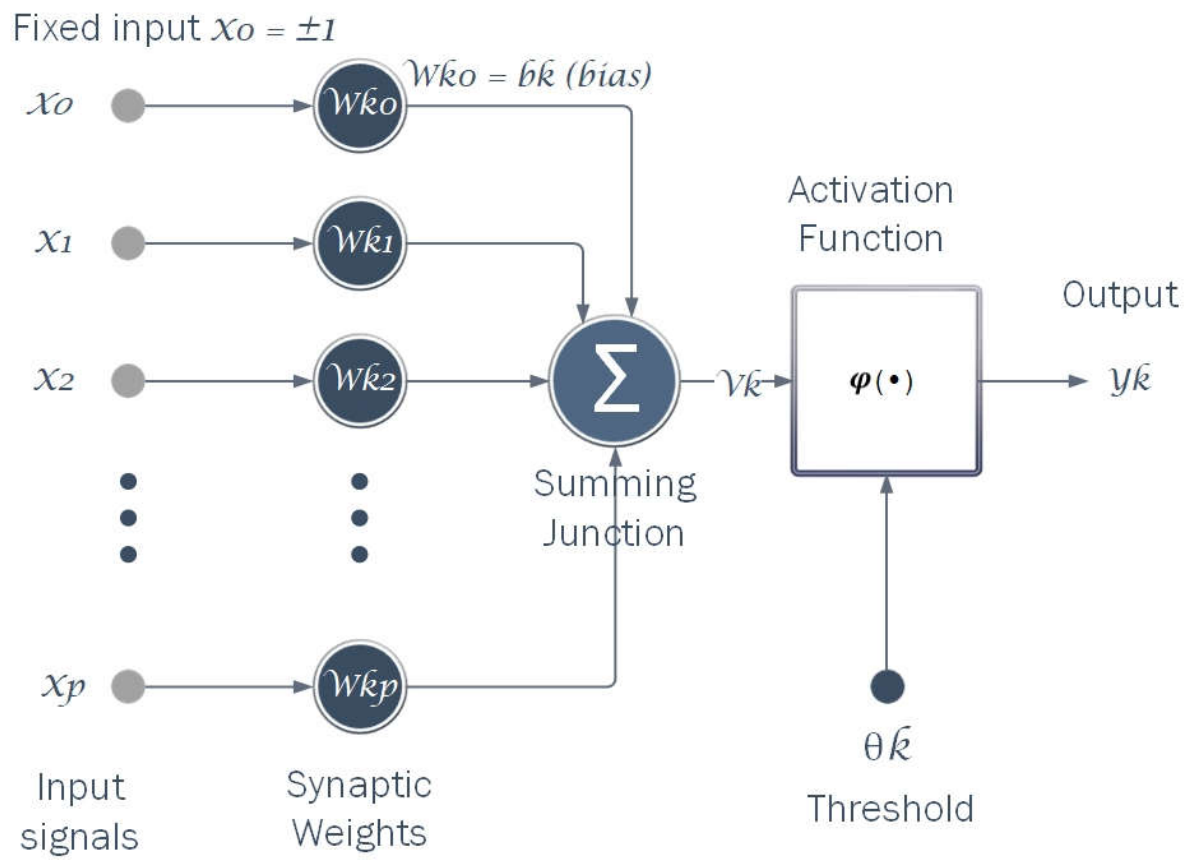

Figure 2. ANN Illustration

The general formula used as Activation Function (AF) is Sigmoid, TanH, and Linear Rectifier. In this example the sigmoid used is as follows.

$\sigma(\mathrm{z})=\frac{1}{1+\mathrm{e}^{-\mathrm{z}}}$ 
In nodes in the output, layer represent the existing classes. The proceeds of $\mathrm{AF}$ is a representation of the value of closeness to a class. The highest value will be used as the class determiner. The range of sigmoid function is 0 to 1 . Each result of AF is the final output or it can also be the input value for the next neuron. The learning process can be illustrated if the input value is $a, b$ and $c$, then the output must be $m$. And if the input value is $d, a$, and $f$, then the output must be $\mathrm{k}$. And so on until the whole iteration is reached. In the learning process, when the desired results are not appropriate, it is necessary to update the weight update is as follows.

$\mathbf{w}_{\mathrm{i}}=\mathbf{w}_{\mathrm{i}}+\operatorname{error}{ }^{*} \boldsymbol{\alpha}_{\mathrm{i}} * \boldsymbol{\mu}$

This learning process is similar to regression. If the regression is looking for coefficient value at the time of learning, the neural network looks for the weight value [18].

\subsubsection{Deep Learning}

Deep Learning is a specific machine learning subfield, in new learning representation of data emphasizing "consecutive" layer learning of representation. Deep Learning permits computational models structured in multiple preparation layers to study data represented by the different extent of abstraction. which is increasingly meaningful. This layered representation is studied through a model called "Neural Network", structured in a literal layer that is stacked one at a time. In fact what we use in Deep Learning is something called artificial neural network (ANN), it is a network that is inspired by a biological neural network used to estimate or estimate a function that can depend on a large number of generally unknown entries [19]
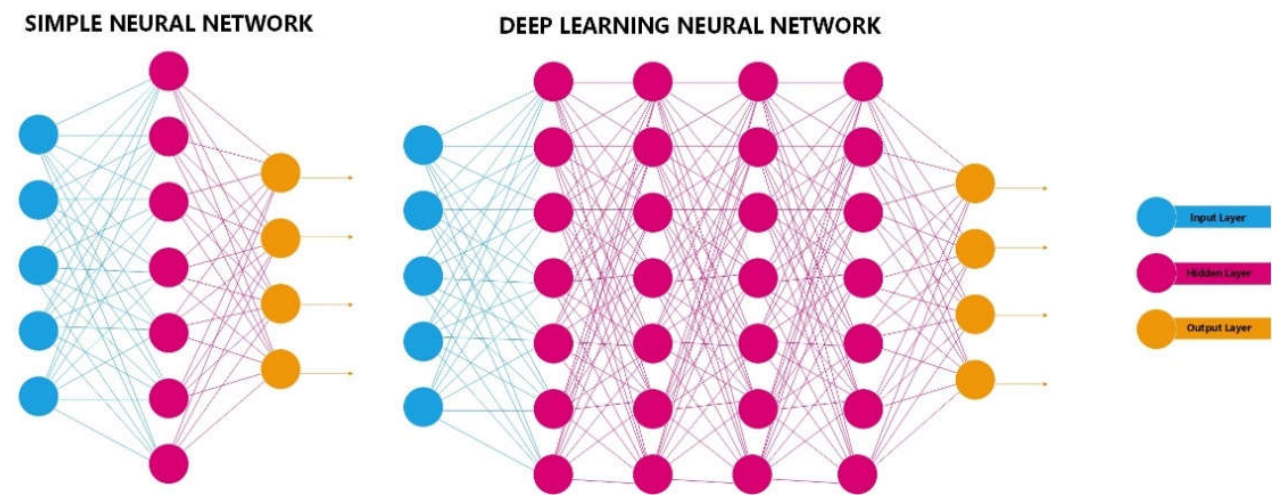

Figure 3. Deep Learning With Many Hidden Layer

Compared to neural networks and multilayer perceptron, Deep Learning is a method that has many hidden layers, each hidden layer represents an increasing level of abstraction. The formula can be formulated as follows:

$f(x) \approx g_{1}\left(g_{2}(\ldots(g n(x)) \ldots)\right)$ 
That from deep learning is how abstraction (hidden layer) is needed and how many nodes (unit layer) have to need of to generate a great learning or design. Alternatively, knowledge time could it be truncated to make momentum this values between 0 and 1 [26]. Momentum may preserve its weight fixed in overall minimum losses. This assist to the backpropagation way when updating the weights. The formulation is as follows [21]:

$v_{i+1}=\mu \nabla \mathbf{L}\left(w_{i}\right)+\mathbf{m} v_{i}$

$w_{i+1}=w i+V_{i+1}$

where wi = in weight at $\mathrm{i}$-th input, $\mu=$ level of learning rate ( 0 to 1$)$ and $\mathrm{m}=$ momentum value ( 0 to 1 ).

\subsection{Performance Measurement}

The matrix ordinary to rate the performance of classification is below[22]:

Table 2. Performance Metric

\begin{tabular}{|c|c|c|c|}
\hline & \multirow{2}{*}{\multicolumn{2}{|c|}{ Predicted Label }} \\
\hline & & & \\
\hline & & Positive & \begin{tabular}{|l|} 
Negative \\
\end{tabular} \\
\hline \multirow{2}{*}{ Actual Label } & Positive & True Positive (TP) & False Negative (FN) \\
\hline & Negative & False Positive (FP) & True Negative (TN) \\
\hline
\end{tabular}

on accuracy and False Postive as performance measurements on method problems, but other researchers [23] [24] [25] discuss that accuracy and False Positive are inadequate and only by make less accurate accuracy in ouput. they recommend accuracy, precision, recall and ROC curves as a preferable performance measurement method.

Table 3. Classification performance measurement

\begin{tabular}{|l|c|}
\hline Measure & Formula \\
\hline Precision & Precision $=\frac{T P}{T P+F P}$ \\
\hline Recall/Sensitivity & Recall/Sensitivity $=\frac{T P}{T P+F N}$ \\
\hline Accuracy & Accuracy $=\frac{T P+T N}{T P+T N+F P+F N}$ \\
\hline F-Measure & $F \quad$ Measure $=\frac{2 \text { Precision Recall }}{\text { Precision }+ \text { Recall }}$ \\
\hline
\end{tabular}


Precision is being the number of positive predictions. Recall or sensitivity is being the number of positive predicted positive label samples. Specificity is being the number of negative label samples prediction as negative. Accuracy is being the number of examples classified correctly exceeding the sum of cases. A part of the feature (FF) is the comparison of the total of features using the classifier to the general number of features in the dataset.

\section{EXPERIMENT AND ANALYSIS}

Figure 4 shows how much MAP data influence between attribute tables and patient data using to prediction in preeclampsia.

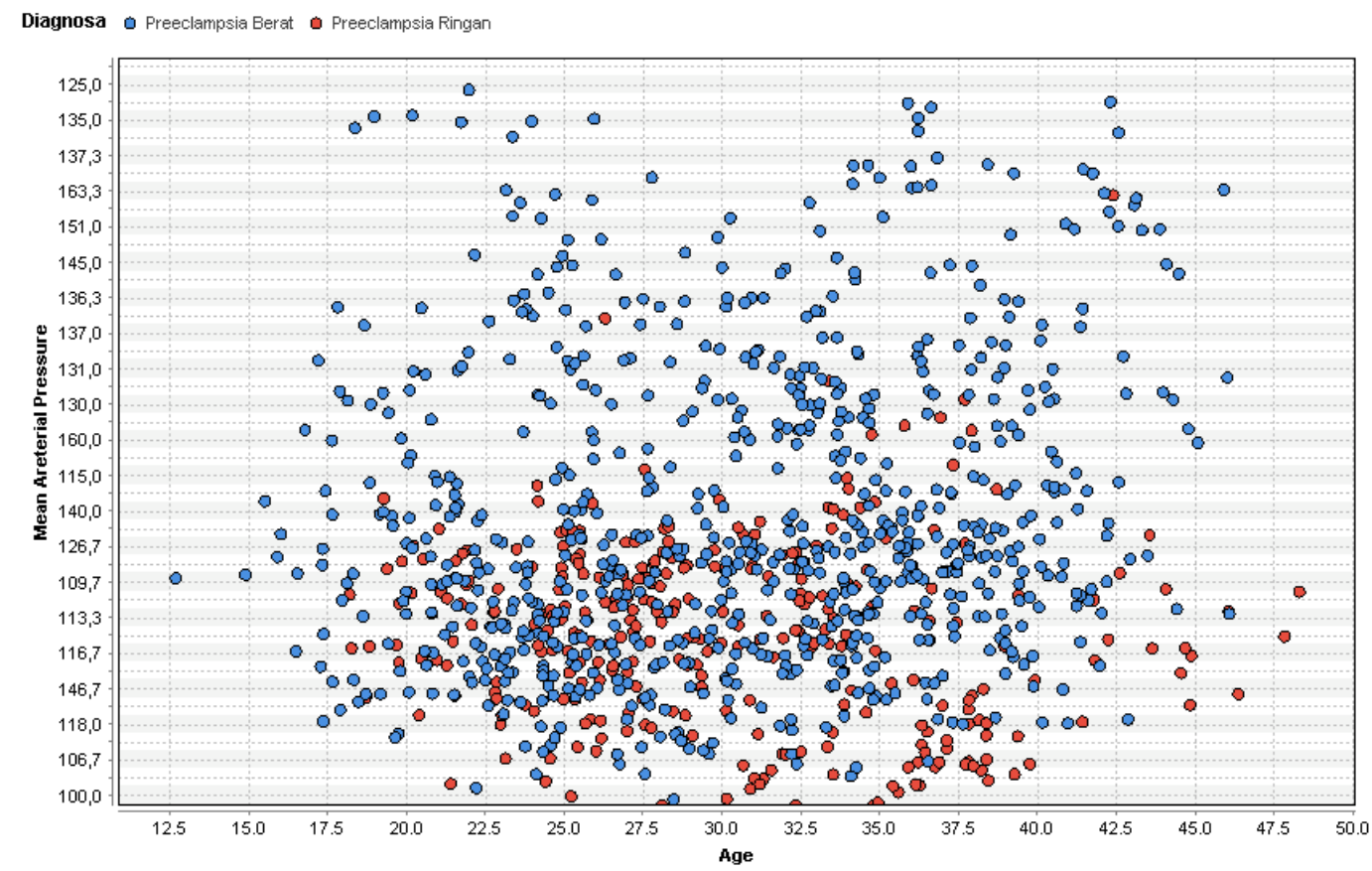

Figure 4. Patient Data Graph

in experiments in the figure upon, we selected in age on the X-Axis and Mean Arterial Pressure in the Y-Axis in take patient data of preeclampsia, that way the scheme show that popular points are found at $<16$ years of age, or the age of pregnant women> 35 years. Mean Arterial Pressure may be an early symptom of preeclampsia if MAP more than $125 \mathrm{mmHg}$ or more can cause severe preeclampsia, hypertension can be observed with an increase in systolic pressure of $30 \mathrm{mmHg}$ above ordinary pressure or a pressure finding in $140 \mathrm{mmHg}$ or more, a rise in diastolic pressure up to $15 \mathrm{mmHg}$ from ordinary pressure or found pressure reaching $90 \mathrm{mmHg}$ or more. Attributes also affecting the learning process is proteinuria that exceeds $0.3 \mathrm{~g} /$ liter or positive 2 in urine 24 hours.

\subsection{Parameter Initialization Particle Swarm Optimization}

The research implementation using PSO consists of certain sizes, specifically the number of particles, the number of iterations, the weight of inertia, the 
speed constants 1 and 2. Jumlah partikel digunakan untuk menentukan banyaknya popsize pada PSO. The number of particles used to specify the number of popsize on the PSO. Then each training data and data testing will be multiply by weight. Adapun nilai parameter pada penelitian ini adalah C1 $=1.0, \mathrm{C} 2=2.0$, Max Generations $=50$, numParticles $=100$, ReportFrequency $=$ 50 , RandomNumberSeed $1=1$ dan Generation $=50$. Determination of this parameter using Weka attribute selection tool PSO Search.

Table 4. Calculate attribute selection PSOSearch

\begin{tabular}{|c|c|c|}
\hline Generasi & merit & Subset \\
\hline 1 & 0.51755 & $4578910111213|9|$ \\
\hline 2 & 0.51755 & $4578910111213|9|$ \\
\hline 3 & 0.51755 & $\begin{array}{l}4578910111213|9| \\
\end{array}$ \\
\hline 4 & 0.51755 & $578910111213|8|$ \\
\hline 5 & 0.51443 & $457810111213|8|$ \\
\hline 6 & 0.51755 & $4578910111213|9|$ \\
\hline 7 & 0.51755 & $4578910111213|9|$ \\
\hline .. & $\ldots$ & $\ldots$ \\
\hline .. & $\ldots$ & $\ldots$. \\
\hline .. & $\ldots$. & $\ldots$ \\
\hline 43 & 0.51755 & $4578910111213|9|$ \\
\hline 44 & 0.51755 & $4578910111213|9|$ \\
\hline 45 & 0.51755 & $4578910111213|9|$ \\
\hline 46 & 0.44233 & $457810111213|8|$ \\
\hline 47 & 0.51755 & $4578910111213|9|$ \\
\hline 48 & 0.51755 & $4578910111213|9|$ \\
\hline 49 & 0.49914 & $457810111213|8|$ \\
\hline 50 & 0.51755 & $4578910111213|9|$ \\
\hline \multicolumn{3}{|c|}{$\begin{array}{l}\text { Best subset found is } 4578910111213 \text { with merit: } 0.5175491051 \\
\text { Attribute Subset Evaluator (supervised, Class (numeric): } 17 \text { Class): } \\
\text { CFS Subset Evaluator } \\
\text { Including locally predictive attributes } \\
\text { Selected attributes: 4,5,7,8,9,10,11,12,13:9 } \\
\text { Maternal_Age } \\
\text { MAP } \\
\text { Classfication_BMI } \\
\text { Previous_PE_Case } \\
\text { Personal_History_of_Hypertension } \\
\text { Personal_Pregnancy_History_of_Hypertension } \\
\text { Diabetes_Pragestasioal } \\
\text { Proteinuria_Reagen_Strip gram/L } \\
\text { Childbirth_Process }\end{array}$} \\
\hline
\end{tabular}




\subsubsection{Feature Selection using Particle Swarm Optimization}

In this study, feature selection results using PSO compared with 7 other popular methods are Naïve Bayes, K-Nearest Neigbour, Decision Tree, Rule Induction, Support Vector Machine, Neural Network and Deep Learning. With Leave One Out in cross validation make each record as sequential test data used in this study. Rapidminer and Weka are used also it research, weka is used to select features from PSO. Rapidminer is used to get good performance.

The table below is the result of feature selection where the operators used are Naïve Bayes, K-NN in $\mathrm{k}=3$, Neural Networks in 100 iterations and the a number of nodes in the hidden layer much in the number of output nodes as well as Deep Learning and libSVM with linear kernels and RBF. The remaining configuration is to use standard parameters on Weka and Rapidminer.

Table 5. Leave-One-Out Cross Validation With Original Data

\begin{tabular}{|c|c|c|c|c|}
\hline \multirow{2}{*}{$\begin{array}{c}\text { Feature } \\
\begin{array}{c}\text { Selection } \\
\text { Original }\end{array}\end{array}$} & \multicolumn{4}{|c|}{ Performance } \\
\cline { 2 - 5 } & Accuracy & Precission & Recall & F-Measure \\
\hline Rule Induction & $84,22 \%$ & $84.89 \%$ & $57,11 \%$ & $68,67 \%$ \\
\hline K-NN & $93,78 \%$ & $89,14 \%$ & $88,48 \%$ & $89,56 \%$ \\
\hline Decision Tree & $78,33 \%$ & $67,16 \%$ & $44,35 \%$ & $51,69 \%$ \\
\hline Naïve Bayes & $92,61 \%$ & $85,45 \%$ & $91,56 \%$ & $89,33 \%$ \\
\hline Deep Learning & $95,12 \%$ & $89,78 \%$ & $90,37 \%$ & $90,37 \%$ \\
\hline SVM & $87,15 \%$ & $79,04 \%$ & $78,18 \%$ & $78,32 \%$ \\
\hline SVM(RBF) & $85.96 \%$ & $83,36 \%$ & $81,11 \%$ & $82,09 \%$ \\
\hline SVM (LINEAR) & $88,10 \%$ & $81,26 \%$ & $79,09 \%$ & $82,79 \%$ \\
\hline Neural Network & $93,18 \%$ & $88,07 \%$ & $88,66 \%$ & $89,42 \%$ \\
\hline
\end{tabular}

The table above shows the accuracy with LOO Validation with the original attribute data indicates that the selection of Deep Learning features is better than the others i.e. $95.12 \%$ on accuracy and F-Measure $90.37 \%$. We resume feature selection experiments on preeclampsia using PSO search and selection of attributes. Of the 17 feature of the preeclampsia, the Particle Swarm Optimization selects obtained 9 of the 17 attributes. Accuracy results are shown in the table below. 
Table 5. Leave-One-Out Cross Validation with Classification results on Particle Swarm Optimization Preeclampsia

\begin{tabular}{|c|c|c|c|c|}
\hline Feature Selection & \multicolumn{4}{|c|}{ Performance } \\
\cline { 2 - 5 } Original (7 Attribute) & Accuracy & Precission & Recall & F-Measure \\
\hline Rule Induction & $84,87 \%$ & $85,25 \%$ & $58,54 \%$ & $69,42 \%$ \\
\hline K-NN & $93,96 \%$ & $89,84 \%$ & $89,56 \%$ & $89,70 \%$ \\
\hline Decision Tree & $77,16 \%$ & $67,16 \%$ & $43,35 \%$ & 52,69 \\
\hline Naïve Bayes & $92,11 \%$ & $83,48 \%$ & $91,14 \%$ & $87,14 \%$ \\
\hline Deep Learning & $95,68 \%$ & $90,16 \%$ & $90,51 \%$ & $90,82 \%$ \\
\hline SVM & $87,65 \%$ & $79,23 \%$ & $78,48 \%$ & $78,86 \%$ \\
\hline SVM(RBF) & $86.06 \%$ & $83,36 \%$ & $81,70 \%$ & $82,39 \%$ \\
\hline SVM (LINEAR) & $88,99 \%$ & $81,76 \%$ & $79,69 \%$ & $83,49 \%$ \\
\hline Neural Network & $93,78 \%$ & $89,27 \%$ & $89,56 \%$ & $89,42 \%$ \\
\hline
\end{tabular}

Feature Selection Using PSO on Preeclampsia Data Set

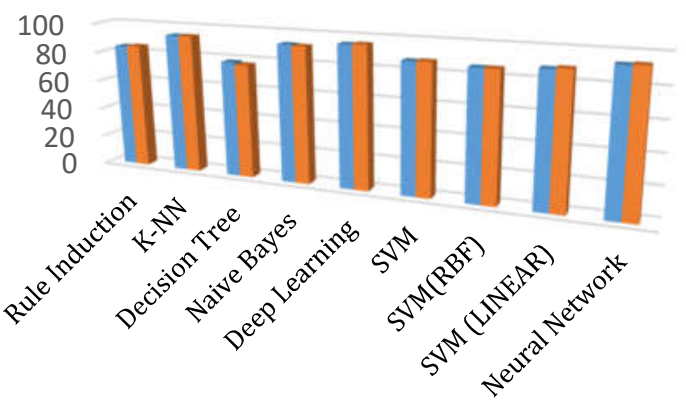

- Accuracy_OriginalData accuracy_PSO

FMeasure Feature Selection Using PSO on Preeclampsia Data Set

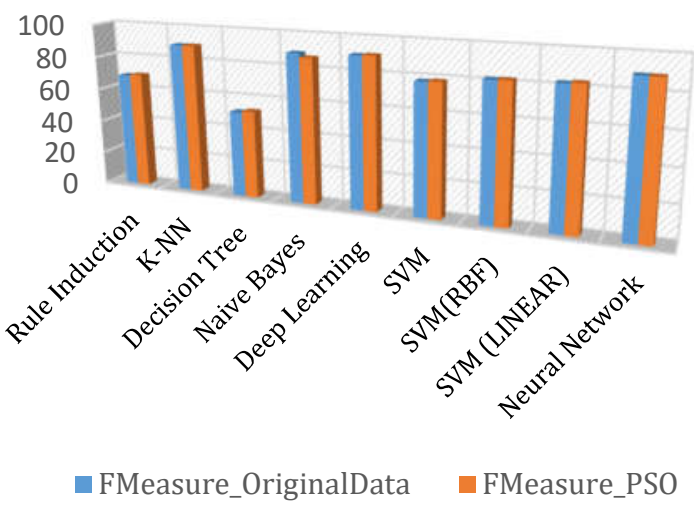

Figure4. Classification results on PSO-reduced Preeclampsia dataset 
The above represents the accuracy of Naïve Bayes, K-Nearest Neighbour, Rule Induction, Decision Tree, Neural Network, Deep Learning, and Support Vector Machine with Leave One Out Cross Validation Methods Comparison of accuracy indicates in the selection of Particle Swarm Optimization features with Deep Learning is better than on the other, accuracy $95.68 \%$ and FMeasure 90.82\%. Particle Swarm Optimization (PSO) has managed to reduce the number of attributes from 17 to 9 , the results show a good performance when compared to the original data. As shown in the above 2 experimental results have relatively steady performance as the total of attributes is only seven attributes. Deep Learning is the only algorithm where its performance some increased from $95.12 \%$ to $95.68 \%$.

\section{CONCLUSION}

We have implemented Particle Swarm Optimization to preeclampsia data to bring down the total of features. The PSO has indicated well-done outcome in the selection of the at the very significant features for same time maintaining the detection value of preeclampsia. As subtraction of feature reduction, the PSO was able to bring down the total of features that initially had 17 attributes to 9 attributes because PSO can reduce the number of attributes because in this optimization method attention to every so dynamic speed on each particle tested and these particles tend to go to better search area during search optimization process take place, so will get the result of good accuracy. And this PSO method is an optimization algorithm that optimizes the attribute weights. While population-based searches are initialized with random populations often referred to as particles.

As subtraction of PSO classification showing tends to show stable results, in my experiment, I applied Machine Learning algorithms namely Naive Bayes, Decision Tree (DT), K-Nearest Neighbor (K-NN), Rule Induction ( RI), SVM, Neural Network (NN) and Deep Learning (DL) into 2 different sets of preeclampsia sets (original dataset and particle swarm optimization Reduced data sets). I set up that Deep Learning excellent 6 different algorithms because in the six algorithms that are often compared to engineering features to get good results, the engineering features need to be tidy on attributes or features and this takes a lot of time and complex systems and understanding about in-depth data and here Deep Learning enables end-to-end framework, simply provide input and output only. and when the application of Deep Learning into the original data accuracy reached $95.12 \%$. yet, the selection algorithm doesn't constantly correct its sensibility, make use of the same classifier Deep Learning in the dataset, PSO-reduced is obtained with little accuracy showing an accuracy of $95.12 \%$ to $95.68 \%$ and the execution eightspeed quicker than the original data 


\section{Acknowledgements}

Haji General Hospital Surabaya, East Java, Indonesia. We Thank You and gladly acknowledge dr. Dwinanto Ananda Muttaqin, SpOG for his donation to the first determination in parameters on preeclampsia dataset and its direction and motivation.

\section{REFERENCES}

[1] Fukui A, Yokota M, Funamizu A, Nakamua R, Fukuhara R, Yamada K, Kimura H, Fukuyama A, Kamoi M, Tanaka K, Mizunuma H. Changes of NK cells in preeclampsia. American journal of reproductive immunology. 2012 Apr 1;67(4):278-86.

[2] Roberts JM, Gammill HS. Preeclampsia: recent insights. Hypertension. 2005 Dec 1;46(6):1243-9.

[3] Wicaksono AP, Badriyah T, Basuki A. Comparison of The DataMining Methods in Predicting The Risk Level of Diabetes. EMITTER International Journal of Engineering Technology. 2016 Aug 3;4(1):164-78.

[4] Fahrudin TM, Syarif I, Barakbah AR. Data Mining Approach for Breast Cancer Patient Recovery. EMITTER International Journal of Engineering Technology. 2017 Jul 23;5(1):36-71.

[5] Moreira MW, Rodrigues JJ, Oliveira AM, Ramos RF, Saleem K. A preeclampsia diagnosis approach using bayesian networks. InCommunications (ICC), 2016 IEEE International Conference on 2016 May 22 (pp. 1-5). IEEE.

[6] Moreira MW, Rodrigues JJ, Oliveira AM, Saleem K, Neto AV. An inference mechanism using bayes-based classifiers in pregnancy care. Ine-Health Networking, Applications and Services (Healthcom), 2016 IEEE 18th International Conference on 2016 Sep 14 (pp. 1-5). IEEE.

[7] Tejera E, Jose areias M, Rodrigues A, Ramoa A, Manuel nieto-villar J, Rebelo I. Artificial neural network for normal, hypertensive, and preeclamptic pregnancy classification using maternal heart rate variability indexes. The Journal of Maternal-Fetal \& Neonatal Medicine. 2011 Sep 1;24(9):1147-51.

[8] Saha S, Biswas S, Acharyya S. Gene selection by sample classification using $k$ nearest neighbor and meta-heuristic algorithms. InAdvanced Computing (IACC), 2016 IEEE 6th International Conference on 2016 Feb 27 (pp. 250-255). IEEE.

[9] Neocleous CK, Anastasopoulos P, Nikolaides KH, Schizas CN, Neokleous KC. Neural networks to estimate the risk for preeclampsia occurrence. In Neural Networks, 2009. IJCNN 2009. International Joint Conference on 2009 Jun 14 (pp. 2221-2225). IEEE.

[10] Moreira MW, Rodrigues JJ, Oliveira AM, Saleem K, Neto AJ. Predicting hypertensive disorders in high-risk pregnancy using the random 
forest approach. InCommunications (ICC), 2017 IEEE International Conference on 2017 May 21 (pp. 1-5). IEEE.

[11] Cheng W, Fang L, Yang L, Zhao H, Wang P, Yan J. Varying coefficient models for analyzing the effects of risk factors on pregnant women's blood pressure. InMachine Learning and Applications (ICMLA), 2014 13th International Conference on 2014 Dec 3 (pp. 5560). IEEE.

[12] Roberts JM, Pearson G, Cutler J, Lindheimer M. Summary of the NHLBI working group on research on hypertension during pregnancy. Hypertension. 2003 Mar 1;41(3):437-45.

[13] Nasional BP, Nasional BP. Laporan pencapaian tujuan pembangunan milenium di indonesia 2011. Meningkatkan Kesehatan Ibu. 2012:53-66.

[14] Rahm E, Do HH. Data cleaning: Problems and current approaches. IEEE Data Eng. Bull.. 2000 Dec;23(4):3-13.

[15] Othman ZA, Bakar AA, Hamdan AR, Omar K, Shuib NL. Agent based preprocessing. InIntelligent and Advanced Systems, 2007. ICIAS 2007. International Conference on 2007 Nov 25 (pp. 219-223). IEEE.

[16] Tjiong AS, Monteiro ST. Feature selection with PSO and kernel methods for hyperspectral classification. InEvolutionary Computation (CEC), 2011 IEEE Congress on 2011 Jun 5 (pp. 17621769). IEEE.

[17] Schuh MA, Angryk RA, Sheppard JW. Evolving Kernel Functions with Particle Swarms and Genetic Programming. InFLAIRS Conference 2012 May 16.

[18] Haykin S, Network N. A comprehensive foundation. Neural networks. $2004 \mathrm{Feb} ; 2(2004): 41$.

[19] Witten IH, Frank E, Hall MA, Pal CJ. Data Mining: Practical machine learning tools and techniques. Morgan Kaufmann; 2016 Oct 1.

[20] Krizhevsky A, Sutskever I, Hinton GE. Imagenet classification with deep convolutional neural networks. InAdvances in neural information processing systems 2012 (pp. 1097-1105).

[21] Zeiler MD, Krishnan D, Taylor GW, Fergus R. Deconvolutional networks. InComputer Vision and Pattern Recognition (CVPR), 2010 IEEE Conference on 2010 Jun 13 (pp. 2528-2535). IEEE.

[22] Jwo DJ, Chang SC. Particle swarm optimization for GPS navigation Kalman filter adaptation. Aircraft Engineering and Aerospace Technology. 2009 Jul 3;81(4):343-52.

[23] Davis J, Goadrich M. The relationship between Precision-Recall and ROC curves. InProceedings of the 23rd international conference on Machine learning 2006 Jun 25 (pp. 233-240). ACM.

[24] Kotsiantis SB, Zaharakis I, Pintelas P. Supervised machine learning: A review of classification techniques. Emerging artificial intelligence applications in computer engineering. 2007 Jun 10;160:324. 
[25] Williams N, Zander S, Armitage G. A preliminary performance comparison of five machine learning algorithms for practical IP traffic flow classification. ACM SIGCOMM Computer Communication Review. 2006 Oct 10;36(5):5-16. 